American Journal of Applied Sciences 9 (8): 1219-1224, 2012

ISSN 1546-9239

(C) 2012 Science Publications

\title{
Pitch Analysis of Ukulele
}

\author{
${ }^{1,2}$ Suphattharachai Chomphan \\ ${ }^{1}$ Department of Electrical Engineering, Faculty of Engineering at Si Racha, \\ Kasetsart University, 199 M.6, Tungsukhla, Si Racha, Chonburi, 20230, Thailand \\ ${ }^{2}$ Center for Advanced Studies in Industrial Technology, Kasetsart University, \\ 50 Ngam Wong Wan Rd, Ladyaow, Chatuchak, Bangkok, 10900, Thailand
}

\begin{abstract}
Problem statement: The ukulele is a trendy instrument in the present day. It is a member of the guitar family of instruments which employs four nylon or gut strings or four courses of strings. However, a statistical analysis of the pitch of this instrument has not been conducted. To analysis pitch or fundamental frequency of its main cords should be performed in an appropriate way. This study brings about its effective sound synthesis which is an important issue in the future. Approach: An efficient technique for the analysis of the fundamental frequency (F0) of the human speech had been applied to the analysis of main cords of the ukulele. The autocorrelation-based technique was used with the signal waveform to extract the optimal period or pitch for the corresponding analyzed frame in time domain. Then the corresponding fundamental frequency was calculated in the frequency domain. Results: The 21 main cords were chosen in the study. It had been seen that the existing fundamental frequency values were varied from one to three values. The value was ranging from $65.42 \mathrm{~Hz}-329.93 \mathrm{~Hz}$. Conclusion: By using the analysis technique of fundamental frequency of the human speech, the output frequencies of all main cords can be extracted. It can be empirically seen that they have their unique values from each others.
\end{abstract}

Key words: Ukulele, pitch analysis, fundamental frequency, analysis of fundamental frequency, autocorrelation-based technique

\section{INTRODUCTION}

The ukulele, a member of the guitar family of instruments is presently popular in the teenager society. It employs four nylon or gut strings or four courses of strings. Its tone and volume varies with its size and construction. Ukuleles come basically in four sizes: soprano, concert, tenor and baritone. The soprano is the standard size and is selected in this study.

F0 estimation for the human speech, also referred to as pitch detection, has been a popular research topic for several years and is still being investigated presently (Seresangtakul and Takara, 2003). The basic problem is to extract the F0 from a human speech signal, which is usually the lowest frequency component. In a periodic waveform, most partials are harmonically related, meaning that the frequency of most of the partials are related to the frequency of the lowest partial by a small whole number ratio. The frequency of this lowest partial is F0 of the waveform. An example of F0 contour is presented in logarithmic scale in Fig. 1 (Ravichandran and Samy, 2006; Chomphan and Kobayashi, 2008; 2009; Chomphan, 2010a; 2010b).

By applying the autocorrelation-based approach, the F0 can be extracted from the signal waveform in the periodical interval (Chomphan and Kobayashi, 2008; 2009). This approach is applied to the analysis of pitch of the ukulele with the same procedure.

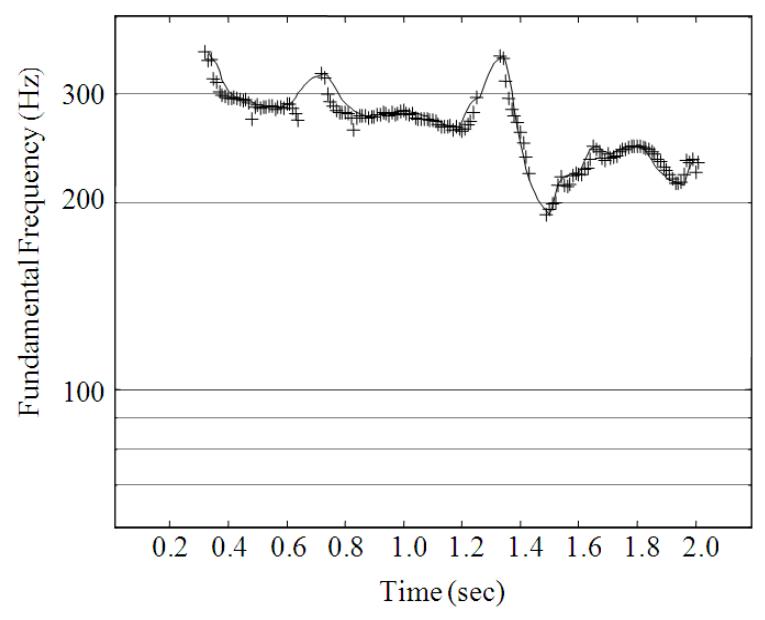

Fig. 1: An example of F0 contours with a trend line in a logarithmic scale

The twenty-one main cords of A, A7, Am, B, B7, Bm, C, C7, Cm, D, D7, Dm, E, E7, Em, F, F7, Fm, G, $\mathrm{G} 7$ and $\mathrm{Gm}$, are chosen to be analyzed. The F0 values of all cords are calculated by using the autocorrelationbased approach. Their existing fundamental frequency values are compared among all selected cords. 


\section{MATERIALS AND METHODS}

Experimental design: Figure 2 shows the procedure of the experiment. The first step is to construct the sound database of twenty-one cords including A, A7, Am, B, B7, Bm, C, C7, Cm, D, D7, Dm, E, E7, Em, F, F7, Fm, G, G7 and Gm. Each cord contains 50 sound samples.

The second step is the autocorrelation-based F0 extraction. Thereafter the F0 trajectory of each sample is analyzed in the last step. The F0 values are grouped into several levels. All sound samples are subsequently averaged. Therefore the F0 values are summarized for each cord.

\section{RESULTS}

As for the ukulele sound material, the database of twenty-one cords including A, A7, Am, B, B7, Bm, C, C7, Cm, D, D7, Dm, E, E7, Em, F, F7, Fm, G, G7 and $\mathrm{Gm}$ is constructed. Each main cord contains 50 sound samples. By using the experimental procedure, the examples of the F0 contours of all cords are depicted in Fig. 3-23.

The averaged existing F0 values of each cord are presented in Table 1 (Mixdorff and Fujisaki, 1997; Fujisaki and Sudo, 1971; Chomphan and Kobayashi, 2007; Chompun et al., 2000). The differences of the F0 values can be seen from this table.

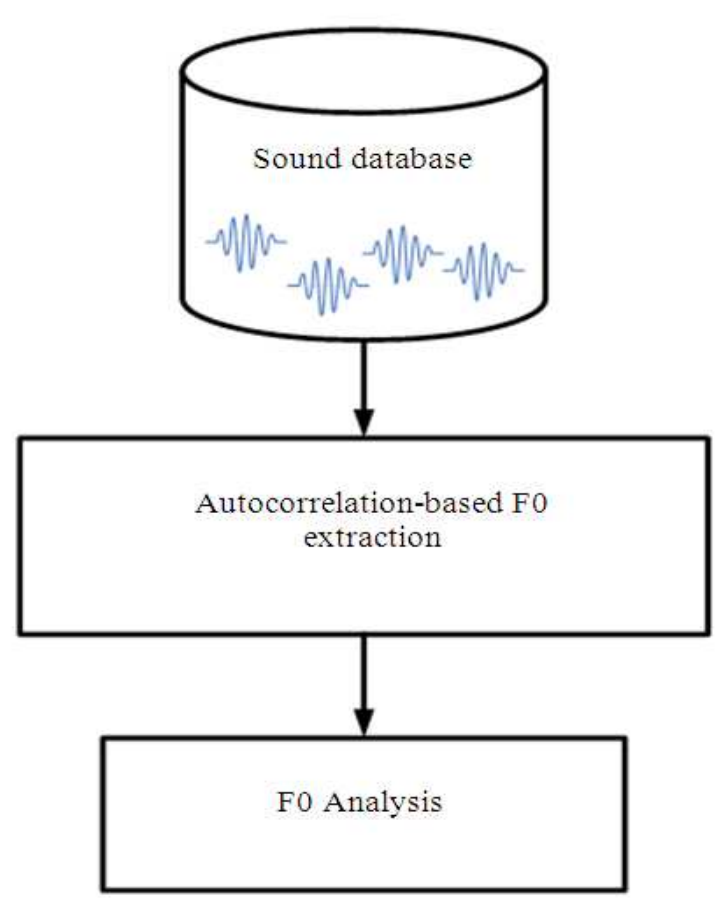

Fig. 2: The flow chart indicating the procedures in the experiment

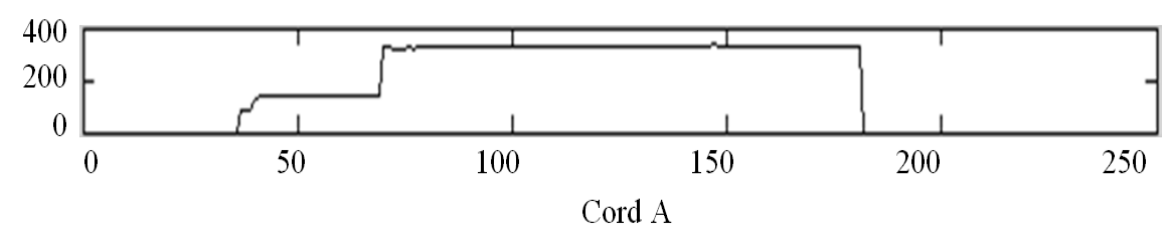

Fig. 3: An example of the F0 contour of cord A

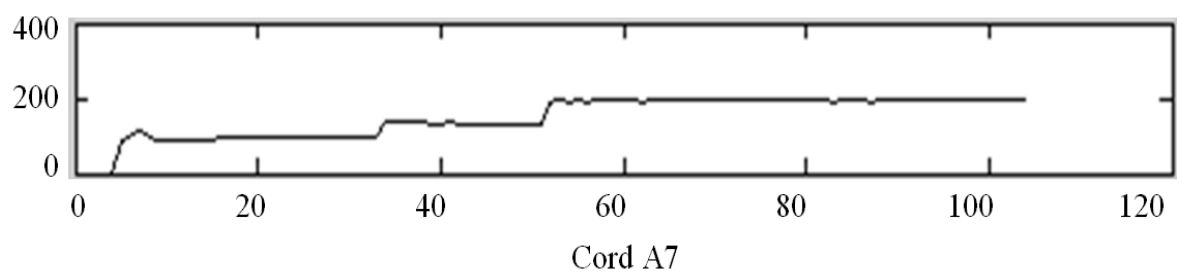

Fig. 4: An example of the F0 contour of cord A7

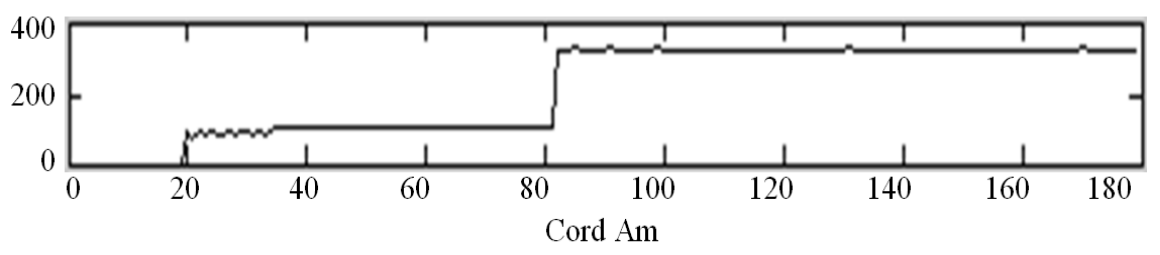

Fig. 5: An example of the F0 contour of cord Am 
Am. J. Applied Sci., 9 (8): 1219-1224, 2012

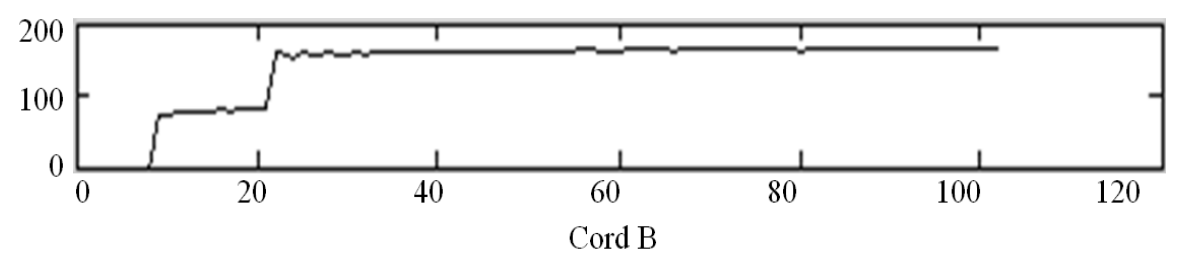

Fig. 6: An example of the F0 contour of cord B

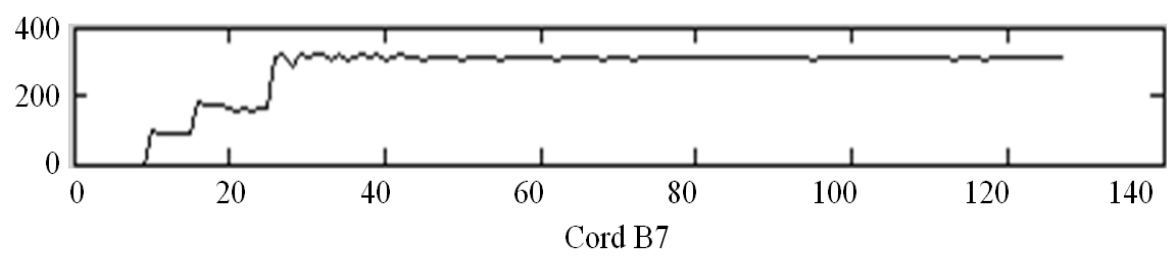

Fig. 7: An example of the F0 contour of cord B7

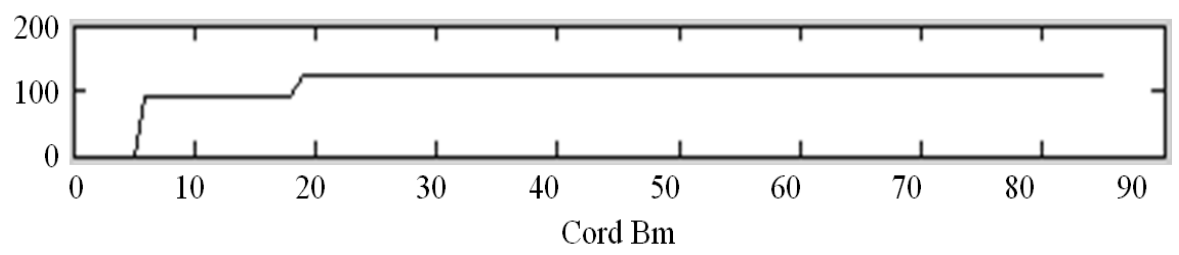

Fig. 8: An example of the F0 contour of cord Bm

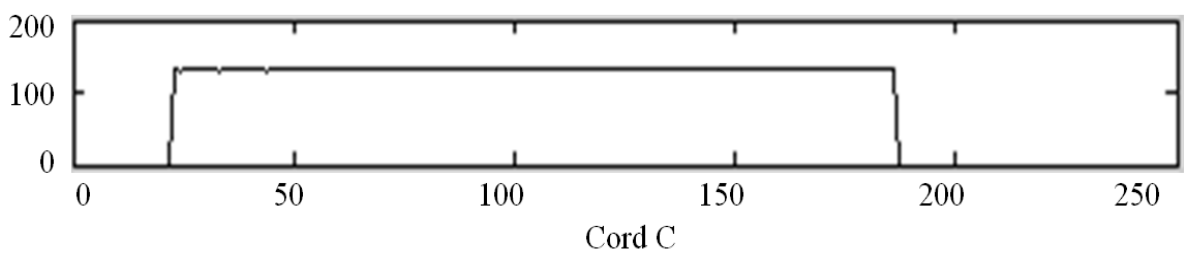

Fig. 9: An example of the F0 contour of cord C

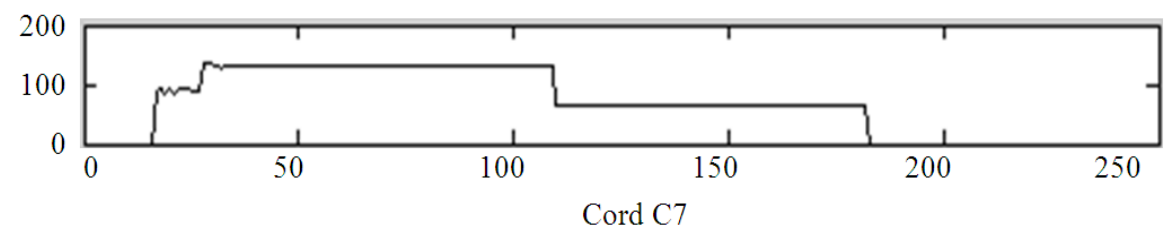

Fig. 10: An example of the F0 contour of cord C7

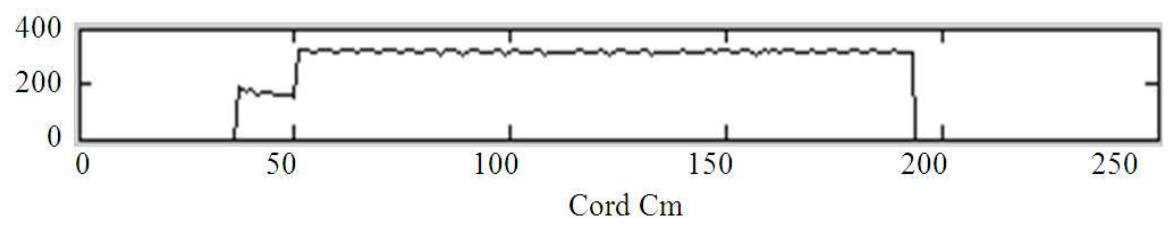

Fig. 11: An example of the F0 contour of cord Cm 
Am. J. Applied Sci., 9 (8): 1219-1224, 2012

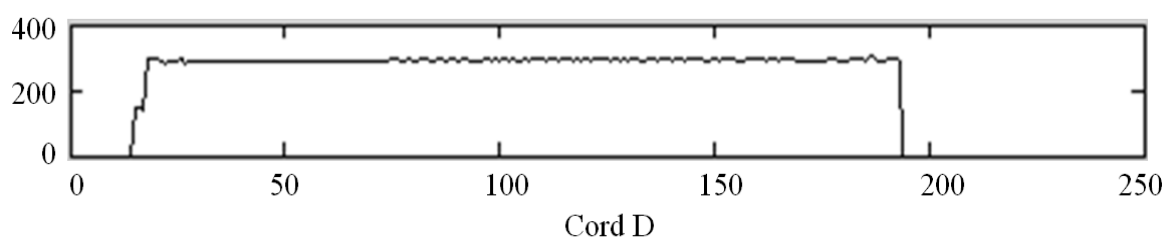

Fig. 12: An example of the F0 contour of cord D

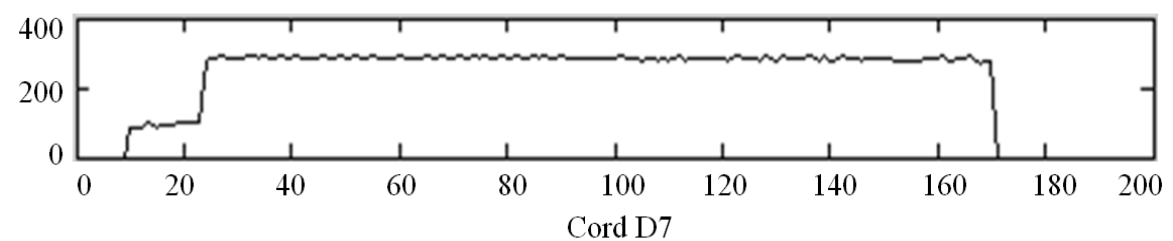

Fig. 13: An example of the F0 contour of cord D7

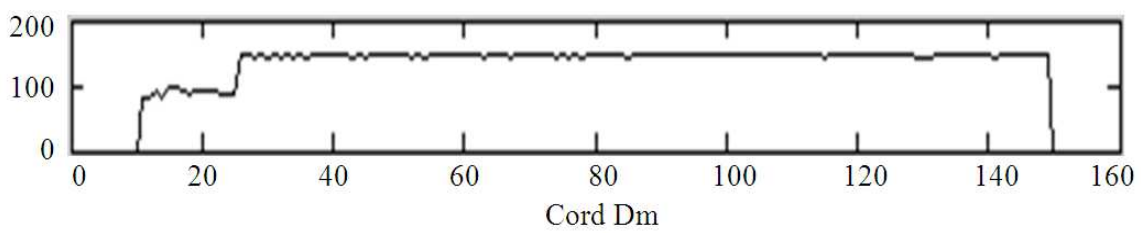

Fig. 14: An example of the F0 contour of cord Dm

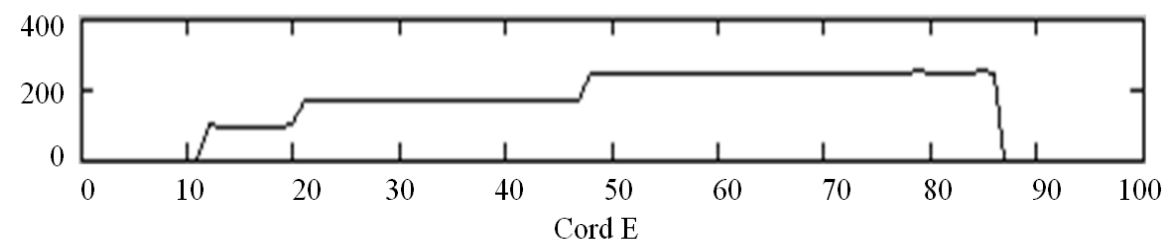

Fig. 15: An example of the F0 contour of cord E

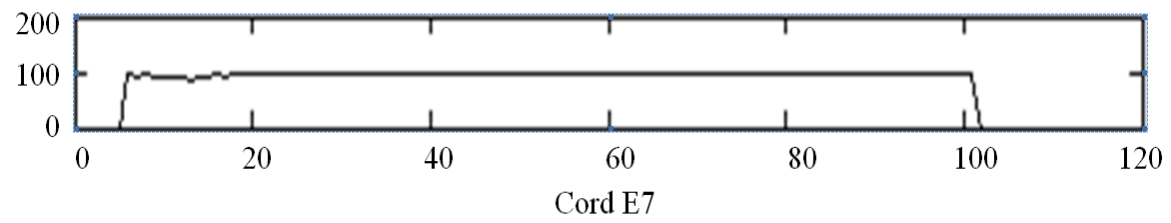

Fig. 16: An example of the F0 contour of cord E7

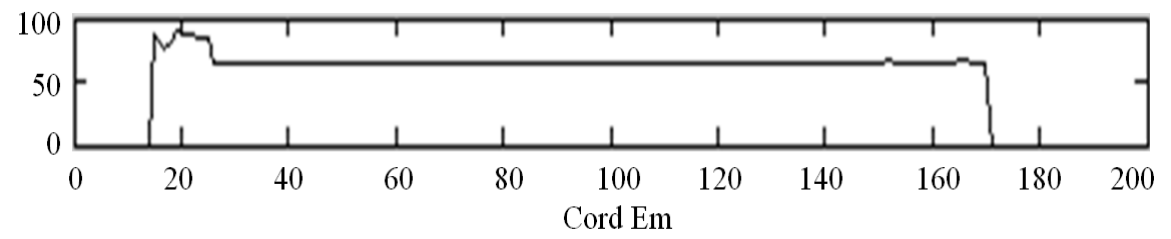

Fig. 17: An example of the F0 contour of cord Em 
Am. J. Applied Sci., 9 (8): 1219-1224, 2012

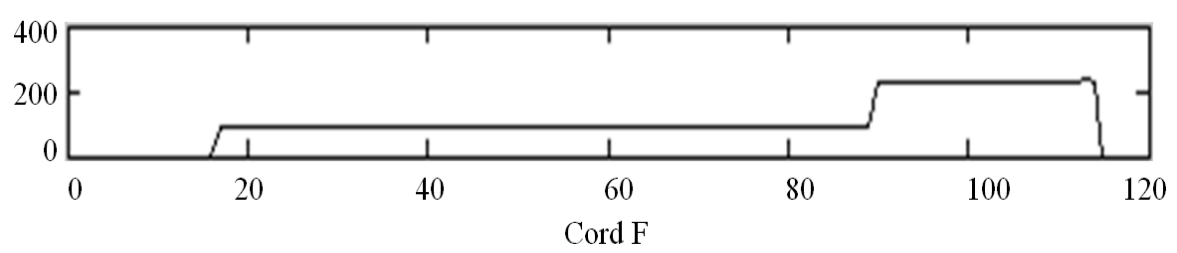

Fig. 18: An example of the F0 contour of cord F

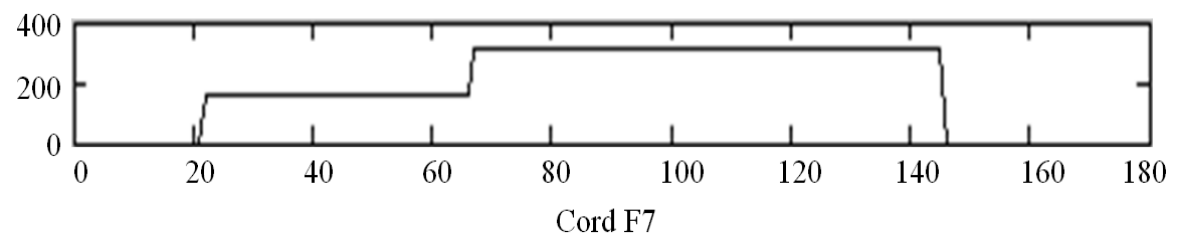

Fig. 19: An example of the F0 contour of cord F7

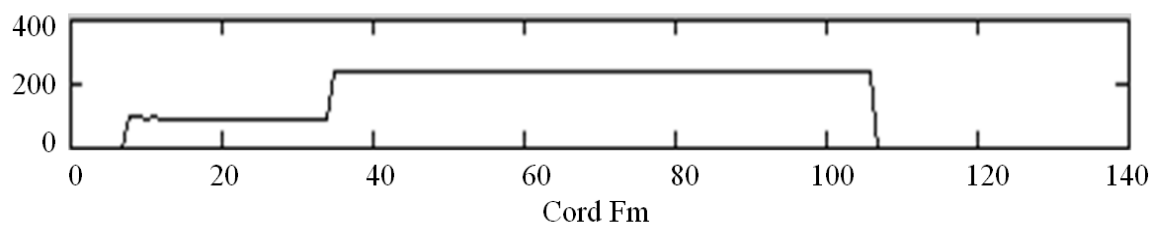

Fig. 20: An example of the F0 contour of cord Fm

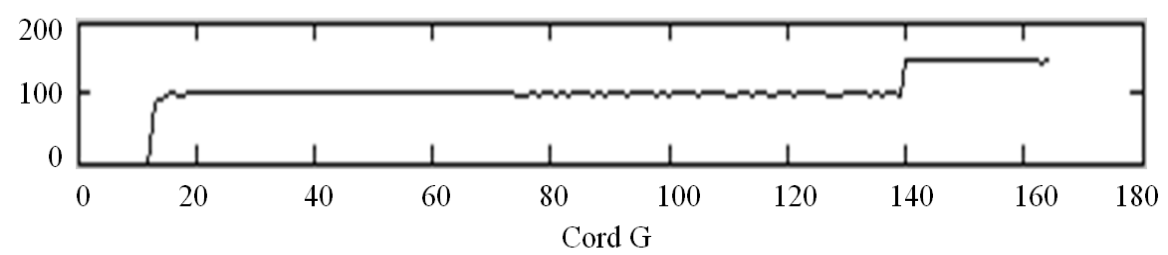

Fig. 21: An example of the F0 contour of cord G

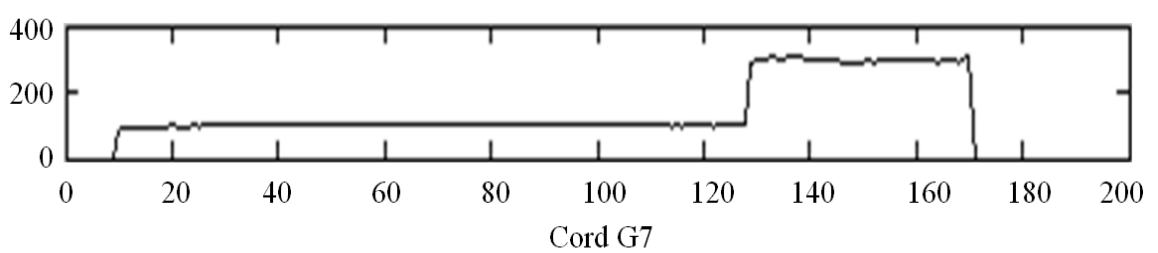

Fig. 22: An example of the F0 contour of cord G7

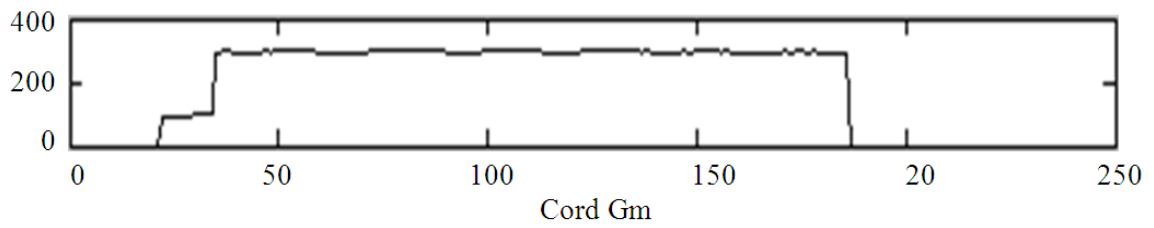

Fig. 23: An example of the F0 contour of cord Gm 
Am. J. Applied Sci., 9 (8): 1219-1224, 2012

Table 1: Averaged existing F0 values of all main cords of ukulele

\begin{tabular}{lrll} 
& Averaged F0 (Hz) & & \\
Cord & 1st value & 2nd value & 3rd value \\
\hline A & 165.66 & 329.93 & \\
A7 & 92.48 & 132.78 & 194.40 \\
Am & 108.87 & 328.10 & \\
B & 75.31 & 161.13 & 294.66 \\
B7 & 88.58 & 163.30 & \\
Bm & 91.82 & 123.60 & \\
C & 130.82 & & \\
C7 & 85.76 & 130.41 & \\
Cm & 152.66 & 314.35 & \\
D & 287.56 & & \\
D7 & 112.42 & 285.46 & \\
Dm & 90.87 & 147.99 & \\
E & 92.68 & 164.10 & \\
E7 & 91.17 & & \\
Em & 66.93 & 246.18 \\
F & 88.24 & 311.11 & \\
F7 & 146.45 & 240.55 & \\
Fm & 87.73 & 260.49 & \\
G & 97.54 & 294.72 & \\
G7 & 96.98 & 295.46 & \\
Gm & 99.61 & & \\
\hline
\end{tabular}

\section{DISCUSSION}

From the experimental results of Table 1, it can be seen that the cords with 3 values of F0 are cord A7, B7, $\mathrm{C} 7$ and E. Moreover, the cords with a value of F0 are cord C, D, E7 and Em, meanwhile the other cords contain a couple of values of F0. It can also be noticed that there is no cord that contains more than three values of F0. On the other hand, each cord contains at least a value of F0. It should be noted that most of the consecutive values of F0 are ranged from the minimal value to the maximal value.

\section{CONCLUSION}

This study presents an analysis of pitch of ukulele which is a popular member of the guitar family of instruments in the present day. A group of pitches or the corresponding values of F0 are analyzed for all main twenty one cords. It can be concluded that there is no cord that contains more than three values of $\mathrm{F} 0$ and each cord contains at least a value of F0. Moreover, most of the consecutive values of $\mathrm{F} 0$ are ranged from the minimal value to the maximal value.

\section{ACKNOWLEDGEMENT}

The researcher is grateful to Kasetsart University for the research scholarship through the Center for Advanced Studies in Industrial Technology.

\section{REFERENCES}

Chomphan, S. and T. Kobayashi, 2007. Implementation and evaluation of an HMM-based Thai speech synthesis system. Proceedings of the 8th Annual Conference of the International Speech Communication Association, Aug. 27-31, ISCA, Antwerp, Belgium, pp: 2849-2852.
Chomphan, S. and T. Kobayashi, 2008. Tone correctness improvement in speaker dependent HMM-based Thai speech synthesis. Speech Commun., 50: 392-404. DOI: 10.1016/j.specom.2007.12.002

Chomphan, S. and T. Kobayashi, 2009. Tone correctness improvement in speaker-independent average-voice-based Thai speech synthesis. Speech Commun., 51: 330-343. DOI: 10.1016/j.specom.2008.10.003

Chomphan, S., 2010a. Analytical study on fundamental Not searchfrequency contours of Thai expressive speech using Fujisaki's model. J. Comput. Sci., 6: 36-42. DOI: $10.3844 /$ jcssp.2010.36.42

Chomphan, S., 2010b. Fujisaki's model of fundamental frequency contours for thai dialects. J. Comput. Sci., 6: 1263-1271. DOI: 10.3844/jcssp.2010.1263.1271

Chompun, S., S. Jitapunkul, D. Tancharoen and T. Srithanasan, 2000. Thai speech compression using CS-ACELP coder based on ITU G.729 standard. Proceedings of the 4th Symposium on Natural Language Processing, May 10-12, NECTEC, Chiangmai, Thailand, pp: 1-5.

Fujisaki, H. and H. Sudo, 1971. A model for the generation of fundamental frequency contours of Japanese word accent. J. Acoust. Soc. Jap., 57: 445-452.

Mixdorff, H. and H. Fujisaki, 1997. Automated quantitative analysis of F0 contours of utterances from a German ToBI-labeled speech database. Proceedings of the 5th European Conference on Speech Communication and Technology, Sep. 2225, Rhodes, Greece, pp: 187-190. http://www.iscaspeech.org/archive/eurospeech_1997/e97_0187.html

Ravichandran, T. and K.D. Samy, 2006. Performance Enhancement on Voice using VAD Algorithm and Cepstral Analysis. J. Comput. Sci., 2: 835-840. DOI: 10.3844 /jcssp.2006.835.840

Seresangtakul, P. and T. Takara, 2003. A generative model of fundamental frequency contours for polysyllabic words of Thai tones. Proceedings of the IEEE International Conference on Acoustics, Speech and Signal Processing, Apr. 6-10, IEEE Xplore Press, Japan, pp: I-452-I-455. DOI: 10.1109/ICASSP.2003.1198815 